\title{
Ascertainment and Verification of End-Stage Renal Disease and End-Stage Liver Disease in the North American AIDS Cohort Collaboration on Research and Design
}

\author{
Mari M. Kitahata, ${ }^{1,2}$ Daniel R. Drozd, ${ }^{1,2}$ Heidi M. Crane, ${ }^{1,2}$ Stephen E. Van Rompaey, \\ Keri N. Althoff, ${ }^{3}$ Stephen J. Gange, ${ }^{3}$ Marina B. Klein, ${ }^{4}$ Gregory M. Lucas, ${ }^{5}$ \\ Alison G. Abraham, ${ }^{3}$ Vincent Lo Re III, ${ }^{6}$ Justin McReynolds, ${ }^{7}$ William B. Lober, ${ }^{7,8}$ \\ Adell Mendes, ${ }^{3}$ Sharada P. Modur, ${ }^{3}$ Yuezhou Jing, ${ }^{3}$ Elizabeth J. Morton, ${ }^{2}$ \\ Margaret A. Griffith, ${ }^{2}$ Aimee M. Freeman, ${ }^{3}$ and Richard D. Moore ${ }^{3,5}$

\footnotetext{
${ }^{1}$ Division of Allergy and Infectious Diseases, Department of Medicine, University of Washington, Seattle, WA 98109, USA

${ }^{2}$ Center for AIDS Research, University of Washington, Seattle, WA 98104, USA

${ }^{3}$ Bloomberg School of Public Health, Johns Hopkins University, Baltimore, MD 21205, USA

${ }^{4}$ Division of Infectious Diseases Chronic Viral Illness Service, Department of Medicine, McGill University, Montreal, QC, Canada

${ }^{5}$ Department of Medicine, Johns Hopkins University, Baltimore, MD 21287, USA

${ }^{6}$ Center for Clinical Epidemiology and Biostatistics, University of Pennsylvania School of Medicine, Philadelphia VA Medical Center, Philadelphia, PA 19104, USA
} \\ ${ }^{7}$ Medical Education and Biomedical Informatics, School of Medicine, University of Washington, Seattle, WA 98109, USA \\ ${ }^{8}$ Biobehavioral Nursing and Health Systems, Department of Nursing, University of Washington, Seattle, WA 98195, USA
}

Correspondence should be addressed to Mari M. Kitahata; kitahata@uw.edu

Received 15 December 2014; Accepted 16 January 2015

Academic Editor: Glenda Gray

Copyright ( $) 2015$ Mari M. Kitahata et al. This is an open access article distributed under the Creative Commons Attribution License, which permits unrestricted use, distribution, and reproduction in any medium, provided the original work is properly cited.

\begin{abstract}
The burden of HIV disease has shifted from traditional AIDS-defining illnesses to serious non-AIDS-defining comorbid conditions Research aimed at improving HIV-related comorbid disease outcomes requires well-defined, verified clinical endpoints. We developed methods to ascertain and verify end-stage renal disease (ESRD) and end-stage liver disease (ESLD) and validated screening algorithms within the largest HIV cohort collaboration in North America (NA-ACCORD). Individuals who screened positive among all participants in twelve cohorts enrolled between January 1996 and December 2009 underwent medical record review to verify incident ESRD or ESLD using standardized protocols. We randomly sampled 6\% of contributing cohorts to determine the sensitivity, specificity, positive predictive value (PPV), and negative predictive value (NPV) of ESLD and ESRD screening algorithms in a validation subcohort. Among 43,433 patients screened for ESRD, 822 screened positive of which 620 met clinical criteria for ESRD. The algorithm had 100\% sensitivity, 99\% specificity, 82\% PPV, and 100\% NPV for ESRD. Among 41,463 patients screened for ESLD, 2,024 screened positive of which 645 met diagnostic criteria for ESLD. The algorithm had 100\% sensitivity, 95\% specificity, 27\% PPV, and 100\% NPV for ESLD. Our methods proved robust for ascertainment of ESRD and ESLD in persons infected with HIV.
\end{abstract}

\section{Introduction}

Antiretroviral therapy (ART) has transformed HIV infection from a rapidly progressive fatal illness to a manageable chronic disease [1]. However, mortality may remain elevated compared to HIV-negative individuals [2-4] as HIV-infected individuals confront an increasing burden of comorbid conditions commonly seen in the aging general population including malignancies and cardiovascular, renal, and liver diseases [5-14]. Federal US HIV/AIDS policy has prioritized 
the study of these age-related conditions in persons infected with HIV $[15,16]$, yet research on HIV-related comorbid disease has been limited by inconsistent diagnostic criteria, reliance on administrative diagnosis data, and lack of verified, definitive clinical outcomes [10-14, 17-31].

Renal disease is common in HIV-infected individuals and spans a spectrum of severity of illness [32]. End-stage renal disease (ESRD), defined as irreversible kidney damage treated with renal replacement therapy (RRT), represents the most significant and definitive clinical endpoint. Many known risk factors for ESRD including diabetes mellitus [33], hypertension [34], and hepatitis $\mathrm{C}$ virus (HCV) coinfection [35] are more common in HIV-infected individuals. There are no definitive criteria for ascertainment or verification of ESRD in persons with HIV infection. Inferences from previous studies of ESRD have been limited by the use of incomplete laboratory data $[10,11]$, composite endpoints [11, $12,29,31$, and focus on a single center [31] or clinical trial setting [20].

End-stage liver disease (ESLD) is the final and often terminal result of chronic liver disease. ESLD-related deaths have increased as a percentage of total deaths amongst HIVinfected individuals [21]. Prevalence of hepatitis B virus (HBV) [36-38] and hepatitis $\mathrm{C}$ virus (HCV) coinfection $[39,40]$ and alcohol abuse [41, 42], all leading causes of ESLD, are increased in persons infected with HIV. ART reduces progression to liver fibrosis in individuals coinfected with $\mathrm{HCV}[43,44]$ and the advent of highly effective direct acting agents (DAAs) marks the beginning of a new HCV treatment era. However, research aimed at improving liver disease outcomes among HIV-infected individuals requires well-defined, clinical ESLD endpoints.

Previous studies of ESLD have used heterogeneous screening criteria and case definitions and focused on specific subpopulations $[13,14,25,26]$ or patients who have undergone liver biopsy [45], thereby introducing potential selection bias. Both the American Association for the Study of Liver Disease (AASLD) and the European Association for the Study of the Liver (EASL) have published guidelines that define diagnoses consistent with ESLD (ascites, spontaneous bacterial peritonitis (SBP), esophageal/gastric variceal hemorrhage, hepatic encephalopathy, and hepatocellular carcinoma), which rely on the presence of one or more clinical events, physical examination, and laboratory, radiographic, or endoscopic findings. Only one study has examined the utility of screening for ESLD among persons infected with HIV, which was conducted in the Veterans Aging Cohort [46].

The North American AIDS Cohort Collaboration on Research and Design (NA-ACCORD) developed standardized protocols to identify and verify four clinically important outcomes in HIV-infected individuals (e.g., myocardial infarction (MI) [47], malignancies, ESRD, and ESLD) and designed web-based applications to improve the efficiency of endpoint verification. In this study, we examined the accuracy and completeness of novel screening algorithms to identify ESRD and ESLD events using routinely collected clinical data in the large and diverse population of
HIV-infected individuals in NA-ACCORD. We used a casecohort design to rigorously test the discriminatory properties of screening protocols and report on the sensitivity, specificity, and negative predictive value (NPV) and positive predictive value (PPV) of algorithms for identifying ESRD and ESLD events validated through comprehensive medical record review using standardized criteria.

\section{Materials and Methods}

2.1. Study Population. NA-ACCORD is a consortium of HIV cohorts from North America and one of seven regional collaborations of the International Epidemiologic Databases to Evaluate AIDS (IeDEA) supported by the National Institutes of Health. Details on this collaboration have been published previously [48]. Briefly, NA-ACCORD is the largest and most diverse cohort of persons infected with HIV in North America and consists of 25 cohorts that collect data on $>130,000$ $\mathrm{HIV}$-infected individuals from more than 200 clinical sites in the US and Canada. These sites reflect the spectrum of HIV disease in North America and include health maintenance organizations, county hospitals, academic medical centers, and private practices in the US and Canada. Each cohort submits standardized clinical data at scheduled intervals including demographic characteristics, medications, laboratory values, and diagnoses on enrolled participants. All patients enrolled between January 1996 and December 2009 in twelve clinical cohorts were included in this study. Whereas previous studies have relied on administrative ICD-9-CM coded billing data, NA-ACCORD captures clinical diagnoses documented prospectively by the treating clinician in the medical record. These data are transferred securely to the NA-ACCORD Data Management Core (DMC) where they undergo quality control for completeness and accuracy and are combined into a harmonized relational database. The human subject activities of the NA-ACCORD and of each of the participating cohort studies have been reviewed and approved by their respective local institutional review boards.

2.2. Data Collection. The NA-ACCORD DMC developed web-based applications to standardize ESRD and ESLD event verification and data collection across cohorts. The webbased platform facilitates secure access to authorized data and reduces administrative time, thereby reducing costs. Medical record review was performed by or under the supervision of a physician at each cohort. Reviewers were presented with potential ESLD or ESRD cases identified in his/her cohort using the screening algorithms (described below) applied centrally by the NA-ACCORD Epidemiology/Biostatistics Core. Diagnoses, medications, procedures, laboratory test results, and other relevant clinical data for each potential case were prepopulated into the application to increase the efficiency of review. The reviewer answered structured questions to verify or invalidate the potential case using drop-down menus, radio-buttons, and checkboxes to ensure the integrity of the data. Electronic data entry facilitated automated checks for missing data. 
2.3. Outcome Screening and Verification Procedures. Screening and verification criteria were developed by NA-ACCORD ESRD and ESLD Working Groups comprising individuals with clinical and epidemiologic expertise in these areas. Comprehensive review of all available medical records was conducted for each individual who screened positive for ESRD or ESLD to confirm the event using a standardized protocol. In those with no evidence of ESRD or ESLD, the absence of the condition was explicitly recorded.

2.3.1. ESRD Screening Criteria. We identified potential ESRD cases using either diagnosis or laboratory criteria consistent with ESRD in HIV-infected individuals [49] outlined below.

(i) Diagnosis Criteria. Diagnosis criteria include any single clinician-documented diagnostic or procedure code consistent with ESRD (see Appendix).

(ii) Laboratory Criteria. Positive laboratory screening criteria included at least two estimated glomerular filtration rate (eGFR) measurements of $<30 / \mathrm{mL} / \mathrm{min} /$ $1.73 \mathrm{~m}^{2}$ separated by greater than 90 days without an intervening measure $\geq 30 \mathrm{~mL} / \mathrm{min} / 1.73 \mathrm{~m}^{2}$. eGFR was calculated using the Chronic Kidney Disease Epidemiology Collaboration (CKD-EPI) equation which incorporates age, race, and sex [50].

2.3.2. ESRD Verification. Criteria used to confirm ESRD are shown in Table 1. Each potential case identified by diagnosis, procedure, or laboratory criteria listed above underwent validation for ESRD by review of all available medical records using a standardized protocol to confirm evidence of RRT defined as chronic dialysis (hemo- or peritoneal dialysis) of greater than six months duration, arteriovenous fistula (AVF) placement with evidence of dialysis, or renal transplantation. Dialysis delivered temporarily (less than six months) for acute kidney injury in hospitalized individuals was not considered ESRD. Dates of renal transplantation, dialysis initiation or AVF placement, confirmation source, kidney biopsy reports, medication use, and substance use were all abstracted from medical records as part of the validation process and recorded in the centralized web-based data entry application. ESRD date was defined as the earliest confirmed date of RRT.

2.3.3. ESLD Screening Criteria. Criteria for ESLD ascertainment included two noninvasive laboratory-based measures of hepatic fibrosis that have been validated in HIV-infected individuals (aspartate aminotransferase (AST)/platelet ratio index (APRI) [51] and FIB-4 [52]) but have not previously been examined for use as screening criteria in this population. The APRI is comprised of the AST and platelet count, and the FIB-4 combines age, platelet count, alanine aminotransferase (ALT), and AST to create an index. Both measures identify advanced hepatic fibrosis/cirrhosis. Applying cut-offs used in previous studies, a positive screen was defined as having at least two APRI scores $>1.5$ or two FIB- 4 scores $>3.25$, greater than 6 months apart. A positive laboratory screen for impaired hepatic function required two of the following laboratory values: total bilirubin $\geq 0.28 \mathrm{mmol} / \mathrm{L}(\geq 5.0 \mathrm{~mL} / \mathrm{dL})$, albumin $<0.11 \mathrm{mmol} / \mathrm{L}(<2.0 \mathrm{mg} / \mathrm{dL})$, or INR $>1.7$, greater than 6 months apart. Those who screened positive for ESLD met either diagnosis or laboratory-based criteria outlined below.

(i) Diagnosis Criteria. Diagnosis criteria include any single clinician-documented diagnostic or procedure code consistent with ESLD (see Appendix).

(ii) Laboratory and Fibrosis Criteria. In order to meet the laboratory criteria to screen positive, a patient needed to have both

(a) a positive lab-based index for advanced hepatic fibrosis (APRI or FIB-4);

(b) at least one other laboratory abnormality consistent with impaired hepatic function (e.g., total bilirubin, albumin, and INR).

2.3.4. ESLD Verification. Each potential case identified by diagnosis and laboratory criteria above underwent validation for ESLD by review of all available medical records using a standardized protocol to confirm evidence of one the following diagnoses: ascites, SBP, variceal hemorrhage, hepatic encephalopathy, or hepatocellular carcinoma. Confirmation of one of these diagnoses met criteria for verified ESLD based on AASLD and EASL ESLD case definitions (Table 1) and the ESLD date was defined as the earliest confirmed diagnosis date. Confirmation source (e.g., radiographic or endoscopic reports), liver biopsy reports, medication use, and substance use were all abstracted from medical records as part of the validation process and recorded in the centralized web-based data entry application.

2.4. Randomly Selected Subcohort. We performed comprehensive medical record review on a randomly selected sample of $9 \%$ of participants from contributing cohorts, termed the "subcohort" [53], to validate screening algorithms for ESRD and ESLD. Given the large number of participants in NA-ACCORD, medical record review of the entire cohort collaboration was not feasible. Two participating cohorts were unable to complete medical record review and one cohort with a large sample size was only able to complete medical record review for one-half of its selected sample. The final subcohort used to validate ESRD and ESLD screening criteria included 2,415 (6\%) and 2,422 (6\%) participants, respectively.

2.5. Data Analysis. We computed sensitivity, specificity, PPV, and NPV of the screening algorithm for ESRD and ESLD in the subcohort participants. Sensitivity was calculated as the proportion of individuals with verified events who screened positive. Specificity was calculated as the proportion of individuals without verified events who screened negative. PPV was calculated as the proportion of screened-positive individuals with a validated event. NPV was calculated as the proportion of screened-negative individuals without a validated event. For ESRD and ESLD, we conducted sensitivity analysis of the screening criteria by separating the criteria as 
TABLE 1: Verification criteria for end-stage renal disease and end-stage liver disease.

\begin{tabular}{|c|c|}
\hline \multicolumn{2}{|r|}{ Criteria for end-stage renal disease } \\
\hline $\begin{array}{l}\text { Hemodialysis/peritoneal } \\
\text { dialysis }\end{array}$ & $\begin{array}{l}\text { Provider documentation of chronic dialysis }(>6 \mathrm{mos}) \text { in dialysis records, inpatient } \\
\text { notes, outpatient clinic notes, or discharge summaries. }\end{array}$ \\
\hline Kidney transplant & $\begin{array}{l}\text { Provider documentation of kidney transplant in inpatient notes, outpatient clinic } \\
\text { notes, or discharge summaries. }\end{array}$ \\
\hline \multicolumn{2}{|r|}{ Criteria for end-stage liver disease } \\
\hline \multirow{5}{*}{ Ascites } & Abdominal ultrasound report indicating ascites \\
\hline & Abdominal CT report indicating ascites \\
\hline & Abdominal MRI report indicating ascites \\
\hline & Abdominal peritoneal fluid analysis result from paracentesis \\
\hline & $\begin{array}{l}\text { Provider documentation of ascites identified by any procedure listed above without } \\
\text { the corroborating primary radiology or laboratory report }\end{array}$ \\
\hline \multirow{4}{*}{ Variceal hemorrhage } & Esophagogastroduodenoscopy (EGD) report of active variceal bleeding \\
\hline & EGD report of recent variceal bleeding \\
\hline & $\begin{array}{l}\text { EGD report of nonbleeding varices in the setting of acute gastrointestinal bleeding } \\
\text { without other causes identified }\end{array}$ \\
\hline & $\begin{array}{l}\text { Provider documentation of variceal hemorrhage identified by EGD procedure } \\
\text { without corroborating primary EGD report }\end{array}$ \\
\hline \multirow{2}{*}{$\begin{array}{l}\text { Spontaneous bacterial } \\
\text { peritonitis }\end{array}$} & Ascitic fluid culture with bacterial growth \\
\hline & Ascitic fluid absolute neutrophil count $\geq 250$ cells $/ \mathrm{mm}^{3}$ \\
\hline \multirow{9}{*}{ Hepatic encephalopathy } & $\begin{array}{l}\text { Mental confusion consistent with hepatic encephalopathy documented in a } \\
\text { progress note of a patient with known chronic liver disease plus absence of any of } \\
\text { the following conditions: }\end{array}$ \\
\hline & $\begin{array}{l}\text { (i) intracranial lesions, such as subdural hematoma, intracranial bleeding, stroke, } \\
\text { tumor, and abscess }\end{array}$ \\
\hline & (ii) infections, such as meningitis, encephalitis, and intracranial abscess \\
\hline & $\begin{array}{l}\text { (iii) metabolic encephalopathy, such as hypoglycemia, electrolyte imbalance, } \\
\text { anoxia, hypercarbia, and uremia }\end{array}$ \\
\hline & $\begin{array}{l}\text { (iv) hyperammonemia from other causes, such as secondary to } \\
\text { ureterosigmoidostomy and inherited urea cycle disorders }\end{array}$ \\
\hline & $\begin{array}{l}\text { (v) toxic encephalopathy from alcohol intake, such as acute intoxication, alcohol } \\
\text { withdrawal, and Wernicke encephalopathy }\end{array}$ \\
\hline & $\begin{array}{l}\text { (vi) toxic encephalopathy from drugs, such as sedative hypnotics, antidepressants, } \\
\text { antipsychotic agents, and salicylates }\end{array}$ \\
\hline & (vii) organic brain syndrome \\
\hline & (viii) postseizure encephalopathy \\
\hline $\begin{array}{l}\text { Hepatocellular } \\
\text { carcinoma }\end{array}$ & Verified through medical record review and/or cancer registries \\
\hline
\end{tabular}

(a) a diagnosis code or a laboratory value; (b) a diagnosis code with or without a laboratory value; (c) a laboratory value with or without a diagnosis code; or (d) both a diagnosis code and a laboratory value. For ESLD, two additional sensitivity analyses were conducted: (a) limiting the diagnosis criteria to the 3 most commonly used codes (ascites, SBP, or variceal hemorrhage) and (b) determining the utility of including procedure codes (liver transplant, paracentesis, and transjugular intrahepatic portosystemic shunt (TIPS)) in our screening algorithm.

\section{Results}

3.1. Participants' Characteristics. Demographic characteristics of individuals who underwent screening for ESRD, ESLD, and the subcohort are shown in Table 2. The proportion of non-Hispanic black individuals was higher among those who screened positive and had confirmed ESRD compared to those who screened negative. The proportion of individuals coinfected with HBV or HCV was higher among those who screened positive and had confirmed ESLD compared to those who screened negative.

3.2. End-Stage Renal Disease. A total of 43,433 patients from 12 cohorts contributed to the ESRD validation study of which 822 screened positive for ESRD by either diagnosis or laboratory criteria and underwent comprehensive medical record review. Two hundred and eighteen individuals were identified by diagnosis criteria alone, 622 were identified by laboratory criteria alone, and 18 were identified by both 


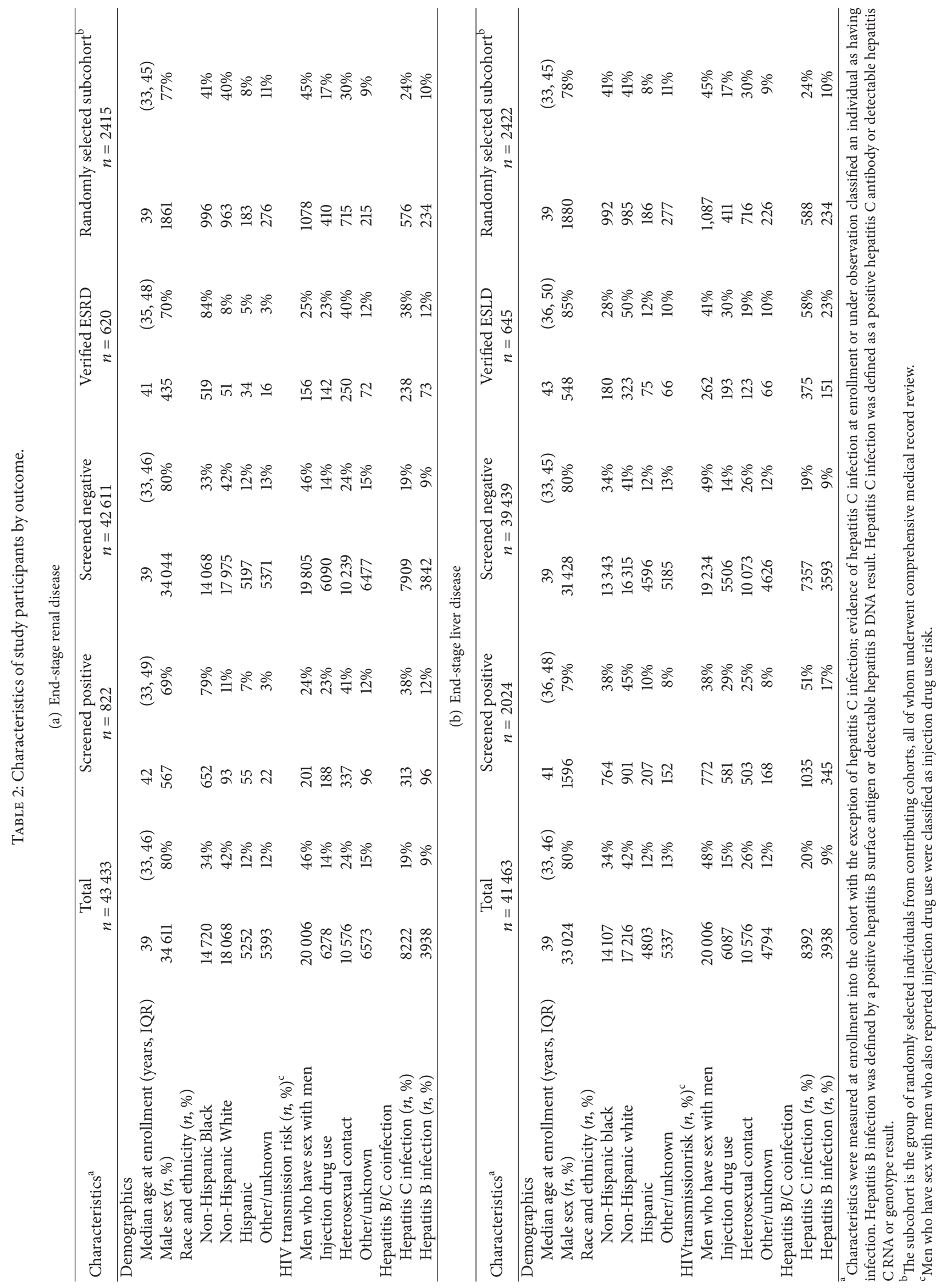


TABLE 3: Sensitivity, specificity, positive predictive value (PPV), and negative predictive value (NPV) of screening algorithms for end-stage renal disease (ESRD) and end-stage liver disease (ESLD) outcomes among participants in the randomly selected subcohort $(n=2,415$ for ESRD and $n=2,422$ for ESLD).

\begin{tabular}{|c|c|c|c|c|c|c|}
\hline Outcome & Screened positive $(n)$ & Verified case $(n)$ & Sensitivity & Specificity & PPV & NPV \\
\hline \multicolumn{7}{|l|}{ End-stage renal disease } \\
\hline Overall (diagnosis OR laboratory) & 76 & 62 & $100 \%$ & $99 \%$ & $82 \%$ & $100 \%$ \\
\hline Diagnosis criteria only & 21 & 17 & $27 \%$ & $100 \%$ & $81 \%$ & $98 \%$ \\
\hline Laboratory criteria only & 58 & 48 & $77 \%$ & $100 \%$ & $83 \%$ & $99 \%$ \\
\hline Diagnosis AND laboratory criteria & 3 & 3 & $5 \%$ & $100 \%$ & $100 \%$ & $98 \%$ \\
\hline \multicolumn{7}{|l|}{ End-stage liver disease } \\
\hline Overall (diagnosis OR laboratory) & 154 & 41 & $100 \%$ & $95 \%$ & $27 \%$ & $100 \%$ \\
\hline Diagnosis criteria only & 135 & 39 & $95 \%$ & $96 \%$ & $29 \%$ & $100 \%$ \\
\hline Laboratory criteria only & 36 & 8 & $20 \%$ & $99 \%$ & $22 \%$ & $99 \%$ \\
\hline Diagnosis AND laboratory criteria & 17 & 6 & $15 \%$ & $100 \%$ & $35 \%$ & $99 \%$ \\
\hline $\begin{array}{l}\text { Diagnosis of ascites, SBP, or variceal } \\
\text { hemorrhage }^{\mathrm{a}}\end{array}$ & 62 & 24 & $59 \%$ & $98 \%$ & $39 \%$ & $99 \%$ \\
\hline
\end{tabular}

a Subgroup of diagnoses used to screen for ESLD.

diagnosis and laboratory criteria. Of the 822 individuals who screened positive overall, 620 met clinical criteria for ESRD. Of the 620 verified cases of ESRD, 159 screened positive by diagnosis criteria, 473 by laboratory criteria, and 12 by both diagnosis and laboratory criteria.

None of the individuals who screened negative for ESRD in the randomly selected subcohort $(n=2,339)$ had verified ESRD. Overall, screening by either diagnosis or laboratory criteria had $100 \%$ sensitivity, 99\% specificity, $82 \% \mathrm{PPV}$, and $100 \%$ NPV. Examined separately, diagnosis criteria were much less sensitive (27\%) than laboratory criteria (77\%), but the specificity and PPV for each was similar to the combined criteria as shown in Table 3. Requiring that both diagnosis and laboratory criteria be met to be classified as screened positive substantially improved the PPV to $100 \%$ though sensitivity was substantially diminished (5\%).

3.3. End-Stage Liver Disease. A total of 41,463 patients from 12 cohorts contributed to the ESLD validation study of which 2,024 screened positive by either diagnosis or laboratory criteria and underwent comprehensive medical record review. Of these, 1,784 individuals were identified by diagnosis criteria alone, 447 by laboratory criteria alone, and 207 by both diagnosis and laboratory criteria. Of the 2,024 individuals who screened positive overall, 645 met diagnostic criteria for ESLD. Of the 645 verified cases identified by either diagnosis or laboratory criteria, 610 were identified by diagnosis criteria alone, 136 by laboratory criteria alone, and 101 by both diagnosis and laboratory criteria.

None of the 2,268 individuals who screened negative for ESLD in the subcohort had verified ESLD. Overall, screening by either diagnosis or laboratory criteria had $100 \%$ sensitivity, 95\% specificity, 27\% PPV, and 100\% NPV as shown in Table 3. Examined separately, diagnosis criteria were highly sensitive (95\%) and specific (96\%), while laboratory criteria were less sensitive (20\%) but highly specific (99\%), and PPV for each were similar (29\% and $22 \%$, resp.). Requiring that both diagnosis and laboratory criteria be met to be classified as screened positive substantially improved the specificity and PPV to $100 \%$ and $35 \%$, respectively, but decreased the sensitivity (15\%).

In sensitivity analyses, 385 (63\%) of the 610 ESLD events identified by diagnosis criteria were identified by a restricted set of diagnoses that included ascites, SBP, or esophageal varices resulting in greater specificity (98\%) and PPV (39\%), but lower sensitivity (59\%). The addition of procedure codes (liver transplant, paracentesis, and TIPS) did not improve the sensitivity of ascertainment over diagnosis and laboratory criteria and, thus, was not included in the overall algorithm.

\section{Discussion}

We developed novel methods to identify and verify ESRD and ESLD that proved robust in the largest and most diverse cohort collaboration of persons infected with HIV in North America, thus being widely applicable to diverse cohorts of $\mathrm{HIV}$-infected individuals to decrease misclassification and improve the validity of inferences from clinical research conducted in this population. Both ESRD and ESLD represent definitive clinical outcomes and add to the collection of adjudicated endpoints available for research within the NAACCORD.

The specificity and PPV of the screening algorithm for ESRD were higher than for ESLD, likely due to the specific nature of RRT and decreased creatinine clearance for ESRD, while the sensitivity of clinical diagnoses alone to identify ESRD was poor. Screening for ESLD relies on less specific markers of liver disease. The inclusion of the APRI and FIB-4 in our laboratory criteria is an important advance as the presence of advanced hepatic fibrosis and cirrhosis necessarily precedes the development of ESLD. To our knowledge, our study is the first to examine these measures for use in ascertainment of ESLD. Combining laboratory markers of advanced liver fibrosis with markers of impaired hepatic function maximized the specificity of ESLD ascertainment, but at the expense of sensitivity. As expected, 
limiting the diagnoses to ascites, SBP, or esophageal varices improved specificity but decreased sensitivity. Procedures that are specific for ESLD, such as liver transplantation and TIPS, were performed infrequently in clinical practice and, thus, did not add to the sensitivity of screening.

4.1. Strengths. Our study has several strengths. It was conducted in the largest, most diverse cohort of persons infected with HIV in the US and Canada making results generalizable across care settings and reflective of the burden of ESLD and ESRD among HIV-infected individuals in North America. Other key strengths include the completeness of inpatient and outpatient clinical data captured from the contributing cohorts, which decreases the likelihood of missing data; the use of standard procedures to harmonize clinical data across sites; systematic centralized ascertainment of potential cases; and standardized protocols for endpoint verification, which minimize misclassification. In addition, we conducted thorough medical record review of a large randomly selected subcohort of individuals to determine the sensitivity, specificity, PPV, and NPV of the screening algorithm for ESRD and ESLD. In order to provide the most rigorous estimates, all calculations were based on the conservative assumption that only those individuals who underwent medical record review were event-free. Comprehensive medical record review conducted for all participants in the randomly selected subcohort facilitates future case-cohort analyses conducted in NAACCORD.

4.2. Limitations. This study has several limitations. First, it is possible that we missed patients within the cohort with ESRD or ESLD. However, thorough review of medical records for over 2,400 cohort participants who were found to be eventfree minimized this risk. Second, we may have misclassified confirmatory events as diagnostic of ESLD or ESRD when, in fact, they were due to other causes. We minimized the risk of misclassification by applying standardized criteria and structured data protocols to define each type of confirmatory events and referring ambiguous or questionable events to the DMC for review.

4.3. CNICS Cohort. We have extended the ESRD and ESLD ascertainment and verification protocols used in NAACCORD to multiple sites in the Centers for AIDS Research Network of Integrated Clinical Systems (CNICS) cohort [54] which includes $>30,000 \mathrm{HIV}$-infected individuals in care from 1995 to the present at eight clinical sites across the US. Applying the same protocol and outcome definitions across NA-ACCORD and CNICS strengthens future collaborations using combined data for analyses. While there is some overlap between the 2 cohorts, each also has independent sites greatly enhancing the potential analytic power. In addition, data collected in the 2 cohorts are complementary. NA-ACCORD provides the very large sample size required to answer key questions related to HIV and ESRD and ESLD that cannot be addressed in smaller cohorts. CNICS provides detailed patient reported data such as the routine measurement of behavioral risk factors not available in other cohorts.
TABLE 4: Diagnoses and procedure codes for ascertainment of ESRD among NA-ACCORD participants.

\begin{tabular}{|c|c|}
\hline \multicolumn{2}{|c|}{ End-stage renal disease } \\
\hline ICD-9-CM codes & Description \\
\hline $581-581.9$ & Nephrotic syndrome \\
\hline $582-582.9$ & Chronic glomerulonephritis \\
\hline $583-583.9$ & $\begin{array}{l}\text { Nephritis and nephropathy, not specified } \\
\text { as acute or chronic }\end{array}$ \\
\hline $585-585.9$ & Chronic kidney disease (CKD) \\
\hline 586 & Renal failure, unspecified \\
\hline $588-588.9$ & $\begin{array}{l}\text { Disorders resulting from impaired renal } \\
\text { function }\end{array}$ \\
\hline $593.71-593.73$ & $\begin{array}{l}\text { Vesicoureteral reflux with reflux } \\
\text { nephropathy }\end{array}$ \\
\hline 593.9 & $\begin{array}{l}\text { Unspecified disorder for kidney and } \\
\text { ureter }\end{array}$ \\
\hline 585.6 & End stage renal disease \\
\hline 792.5 & $\begin{array}{l}\text { Cloudy (hemodialysis) (peritoneal) } \\
\text { dialysis effluent }\end{array}$ \\
\hline V42.0 & $\begin{array}{l}\text { Organ or tissue replaced by transplant } \\
\text { kidney }\end{array}$ \\
\hline V45.1 & Renal dialysis status \\
\hline V56 & $\begin{array}{l}\text { Encounter for dialysis and dialysis } \\
\text { catheter care }\end{array}$ \\
\hline V56.0 & Extracorporeal dialysis \\
\hline V56.1 & $\begin{array}{l}\text { Fitting and adjustment of extracorporeal } \\
\text { dialysis catheter }\end{array}$ \\
\hline V56.2 & $\begin{array}{l}\text { Fitting and adjustment of peritoneal } \\
\text { dialysis catheter }\end{array}$ \\
\hline V56.3 & $\begin{array}{l}\text { Encounter for adequacy testing for } \\
\text { dialysis }\end{array}$ \\
\hline V56.31 & $\begin{array}{l}\text { Encounter for adequacy testing for } \\
\text { hemodialysis }\end{array}$ \\
\hline V56.32 & $\begin{array}{l}\text { Encounter for adequacy testing for } \\
\text { peritoneal dialysis }\end{array}$ \\
\hline V56.8 & Other dialysis \\
\hline
\end{tabular}

\section{Conclusions}

In conclusion, we developed algorithms to identify ESRD and ESLD using routinely collected clinical data and standardized protocols implemented via web-based applications to verify events in the largest and most diverse cohort of persons infected with HIV in North America. Methods developed in NA-ACCORD to identify and confirm ESRD and ESLD are broadly applicable to observational cohort studies and will facilitate research aimed at understanding the underlying mechanism and progression of the changing clinical spectrum of HIV disease.

\section{Appendix}

See Tables 4 and 5. 
TABLE 5: Diagnoses and procedure codes for ascertainment of ESLD among NA-ACCORD participants.

\begin{tabular}{ll}
\hline \multicolumn{2}{c}{ End-stage liver disease } \\
ICD-9-CM codes & Description \\
\hline 789.5 & Ascites \\
$456.0-456.21$ & Esophageal varices \\
$567.0-567.9$ & $\begin{array}{l}\text { Peritonitis in infectious diseases classified } \\
\text { elsewhere }\end{array}$ \\
070.0 & Viral hepatitis A with hepatic coma \\
$070.4-070.49$ & Other specified viral hepatitis with \\
& hepatic coma \\
070.6 & Unspecified viral hepatitis with hepatic \\
570 & coma \\
571 & Acute and subacute necrosis of liver \\
571.5 & Chronic liver disease and cirrhosis \\
571.8 & Cirrhosis of liver without mention of \\
572.2 & alcohol \\
572.3 & Other chronic nonalcoholic liver diseases \\
572.4 & Hepatic encephalopathy \\
782.4 & Portal hypertension \\
V42.7 & Hepatorenal syndrome \\
54.91 & Jaundice, unspecified, not of newborn \\
39.1 & Organ or tissue replaced by transplant \\
& liver \\
& Percutaneous abdominal drainage \\
& Intra-abdominal venous shunt \\
\hline
\end{tabular}

\section{Abbreviations}

AASLD: American Association for the Study of Liver Disease

ACASI: Audio computer-assisted self-interviewing

AIDS: Acquired immunodeficiency syndrome

AKI: Acute kidney injury

ALT: Alanine aminotransferase

APRI: Aspartate aminotransferase/platelet ratio index

ART: Antiretroviral therapy

AST: Aspartate aminotransferase

AV: $\quad$ Arteriovenous

DMC: Data Management Core

EASL: European Association for the Study of the Liver

EGD: Esophagogastroduodenoscopy

eGFR: Estimated glomerular filtration rate

ESLD: End-stage liver disease

ESRD: End-stage renal disease

FIB-4: Fibrosis-4

HCC: Hepatocellular carcinoma

$\mathrm{HCV}$ : Hepatitis $\mathrm{C}$ virus

HD: Hemodialysis

HE: Hepatic encephalopathy

HIV: Human immunodeficiency virus
HIVAN: $\quad$ HIV-associated nephropathy

HMO: Health Maintenance Organization

ICD-9-CM: International Classification of Diseases, 9th Revision, Clinical Modification

IeDEA: International Epidemiologic Databases to Evaluate AIDS

INR: International normalized ratio

MI: $\quad$ Myocardial infarction

NA-ACCORD: North American AIDS Cohort Collaboration on Research and Design

NPV: $\quad$ Negative predictive value

PD: $\quad$ Peritoneal dialysis

PPV: $\quad$ Positive predictive value

PRO: $\quad$ Patient-reported outcome

RRT: Renal replacement therapy

SBP: Spontaneous bacterial peritonitis

SSO: $\quad$ Single sign-on

TIPS: Transjugular intrahepatic portosystemic shunt

US: $\quad$ United States

VACS: Veterans Aging Cohort Study.

\section{Conflict of Interests}

Marina B. Klein has served as a consultant to ViiV Healthcare, AbbVie, and Gilead and has received honoraria for lectures from Janssen Therapeutic, ViiV Healthcare, and Merck and grant support from Shering-Plough. The following authors report no conflict of interests: Mari M. Kitahata, Daniel R. Drozd, Heidi M. Crane, Stephen E. Van Rompaey, Keri N. Althoff, Stephen J. Gange, Gregory M. Lucas, Alison G. Abraham, Vincent Lo Re III, Justin McReynolds, William B. Lober, Adell Mendes, Sharada P. Modur, Yuezhou Jing, Elizabeth J. Morton, Margaret A. Griffith, Aimee M. Freeman, and Richard D. Moore.

\section{Acknowledgments}

This work was supported by Grants U01-AI069918, U01AA013566, U24-AA020794, U01-AA020790, U01-AI31834, U01-AI34989, U01-AI34993, U01-AI34994, U01-AI35004, U01-AI35039, U01-AI35040, U01-AI35041, U01-AI35042, U01-AI35043, U01-AI37613, U01-AI37984, U01-AI38855, U01-AI38858, U01-AI42590, U01-AI68634, U01-AI68636, U01-AI69432, U01-AI69434, U01-DA036935, U01-HD32632, U10-EY08057, U10-EY08052, U10-EY08067, UL1-RR024131, UL1-TR000083, U54-MD007587, G12- MD007583, K01AI071754, K01-AI093197, K23-EY013707, K24-DA00432, K24AI065298, KL2-TR000421, MO1-RR-00052, N02-CP55504, P30-AI027763, P30-AI094189, P30-AI27757, P30-AI27767, P30-AI036219, P30-AI50410, P30-AI54999, P30-MH62246, R01-AA16893, R01-CA165937, R01-DA04334, R01-DA11602, R01-DA12568, R24-AI067039, R56-AI102622, Z01-CP010214, and Z01-CP010176 from the National Institutes of Health, USA; Contract CDC200-2006-18797 from the Centers for Disease Control and Prevention, USA; Contract 90047713 from the Agency for Healthcare Research and Quality, USA; Contract 90051652 from the Health Resources and Services 
Administration, USA; Grants TGF-96118, HCP-97105, CBR-86906, and CBR-94036 from the Canadian Institutes of Health Research, Canada; Canadian Institutes of Health Research (CIHR) New Investigator Award (A. Burchell); Ontario Ministry of Health and Long Term Care; and the Government of Alberta, Canada. Additional support was provided by the Intramural Research Program of the National Cancer Institute and National Institutes of Health. The authors acknowledge NA-ACCORD Collaborating Cohorts and Representatives including AIDS Link to the IntraVenous Experience: Gregory D. Kirk; Adult AIDS Clinical Trials Group Longitudinal Linked Randomized Trials: Constance A. Benson, Ronald J. Bosch, and Ann C. Collier; Fenway Health HIV Cohort: Stephen Boswell, Chris Grasso, and Kenneth H. Mayer; HAART Observational Medical Evaluation and Research: Robert S. Hogg, P. Richard Harrigan, Julio SG Montaner, Angela Cescon, and Hasina Samji; HIV Outpatient Study: John T. Brooks and Kate Buchacz; HIV Research Network: Kelly A. Gebo and Richard D. Moore; Johns Hopkins HIV Clinical Cohort: Richard D. Moore; John T. Carey Special Immunology Unit Patient Care and Research Database, Case Western Reserve University: Benigno Rodriguez; Kaiser Permanente Mid-Atlantic States: Michael A. Horberg; Kaiser Permanente Northern California: Michael J. Silverberg; Longitudinal Study of Ocular Complications of AIDS: Jennifer E. Thorne; Multicenter Hemophilia Cohort Study-II: James J. Goedert; Multicenter AIDS Cohort Study: Lisa P. Jacobson and Gypsyamber D'Souza; Montreal Chest Institute Immunodeficiency Service Cohort: Marina B. Klein; Ontario HIV Treatment Network Cohort Study: Sean B. Rourke, Ann N. Burchell, and Anita R. Rachlis; Retrovirus Research Center, Bayamon Puerto Rico: Robert F. HunterMellado and Angel M. Mayor; Southern Alberta Clinic Cohort: M. John Gill; Studies of the Consequences of the Protease Inhibitor Era: Steven G. Deeks and Jeffrey N. Martin; The Study to Understand the Natural History of HIV/AIDS in the Era of Effective Therapy: Pragna Patel and John T. Brooks; University of Alabama at Birmingham 1917 Clinic Cohort: Michael S. Saag, Michael J. Mugavero, and James Willig; University of North Carolina at Chapel Hill HIV Clinic Cohort: Joseph J. Eron and Sonia Napravnik; University of Washington HIV Cohort: Mari M. Kitahata, Heidi M. Crane, and Daniel R. Drozd; Veterans Aging Cohort Study: Amy C. Justice, Robert Dubrow, and David Fiellin; Vanderbilt-Meharry Centers for AIDS Research Cohort: Timothy R. Sterling, David Haas, Sally Bebawy, and Megan Turner; Women's Interagency HIV Study: Stephen J. Gange and Kathryn Anastos; NA-ACCORD Study Administration; Executive Committee: Richard D. Moore, Michael S. Saag, Stephen J. Gange, Mari M. Kitahata, Keri N. Althoff, Rosemary G. McKaig, Amy C. Justice, and Aimee M. Freeman; Administrative Core: Richard D. Moore, Aimee M. Freeman, and Carol Lent; Data Management Core: Mari M. Kitahata, Stephen E. Van Rompaey, Heidi M. Crane, Daniel R. Drozd, Liz Morton, Justin McReynolds, and William B. Lober; Epidemiology and Biostatistics Core: Stephen J. Gange, Keri N. Althoff, Alison G. Abraham, Bryan Lau, Jinbing Zhang, Yuezhou Jing, Elizabeth Golub, Shari Modur, Cherise Wong, Adell Mendes, and Brenna Hogan.

\section{References}

[1] F. J. Palella Jr., R. K. Baker, A. C. Moorman et al., "Mortality in the highly active antiretroviral therapy era: changing causes of death and disease in the HIV outpatient study," Journal of Acquired Immune Deficiency Syndromes, vol. 43, no. 1, pp. 2734, 2006.

[2] The Antiretroviral Therapy Cohort Collaboration (ART-CC), "Life expectancy of individuals on combination antiretroviral therapy in high-income countries: a collaborative analysis of 14 cohort studies," The Lancet, vol. 372, no. 9635, pp. 293-299, 2008.

[3] N. Lohse, A.-B. E. Hansen, G. Pedersen et al., "Survival of persons with and without HIV infection in Denmark, 19952005," Annals of Internal Medicine, vol. 146, no. 2, pp. 87-95, 2007.

[4] E. Losina, B. R. Schackman, S. N. Sadownik et al., "Racial and sex disparities in life expectancy losses among hiv-infected persons in the united states: impact of risk behavior, late initiation, and early discontinuation of antiretroviral therapy," Clinical Infectious Diseases, vol. 49, no. 10, pp. 1570-1578, 2009.

[5] J. S. Currier, J. D. Lundgren, A. Carr et al., "Epidemiological evidence for cardiovascular disease in HIV-infected patients and relationship to highly active antiretroviral therapy," Circulation, vol. 118, no. 6, pp. e29-e35, 2008.

[6] V. A. Triant, H. Lee, C. Hadigan, and S. K. Grinspoon, "Increased acute myocardial infarction rates and cardiovascular risk factors among patients with human immunodeficiency virus disease," Journal of Clinical Endocrinology and Metabolism, vol. 92, no. 7, pp. 2506-2512, 2007.

[7] C. J. Achenbach, S. R. Cole, M. M. Kitahata et al., "Mortality after cancer diagnosis in HIV-infected individuals treated with antiretroviral therapy," AIDS, vol. 25, no. 5, pp. 691-700, 2011.

[8] G. Lucas, Y. Jing, M. Sulkowski et al., "Hepatitis C viremia and the risk of chronic kidney disease in HIV-infected individuals," The Journal of Infectious Diseases, vol. 208, no. 8, pp. 1240-1249, 2013.

[9] E. L. Yanik, S. Napravnik, S. R. Cole et al., "Incidence and timing of cancer in HIV-infected individuals following initiation of combination antiretroviral therapy," Clinical Infectious Diseases, vol. 57, no. 5, pp. 756-764, 2013.

[10] J. C. Trullas, A. Mocroft, F. Cofan et al., "Dialysis and renal transplantation in HIV-infected patients: a European survey," Journal of Acquired Immune Deficiency Syndromes, vol. 55, no. 5, pp. 582-589, 2010.

[11] L. Ryom, O. Kirk, J. Lundgren et al., "Advanced chronic kidney disease, end-stage renal disease and renal death among HIVpositive individuals in Europe," HIV Medicine, vol. 14, no. 8, pp. 503-508, 2013.

[12] A. I. Choi, R. A. Rodriguez, P. Bacchetti, D. Bertenthal, P. A. Volberding, and A. M. O'Hare, "The impact of HIV on chronic kidney disease outcomes," Kidney International, vol. 72, no. 11, pp. 1380-1387, 2007.

[13] D. Posthouwer, M. Makris, T. T. Yee et al., "Progression to endstage liver disease in patients with inherited bleeding disorders and hepatitis C: an international, multicenter cohort study," Blood, vol. 109, no. 9, pp. 3667-3671, 2007.

[14] J. J. Goedert, M. Elaine Eyster, M. M. Lederman et al., "Endstage liver disease in persons with hemophilia and transfusionassociated infections," Blood, vol. 100, no. 5, pp. 1584-1589, 2002. 
[15] The White House Office of National AIDS Policy, The National HIV/AIDS Strategy, The White House Office of National AIDS Policy, 2010.

[16] NIH Office of AIDS Research, FY 2013 Trans-NIH Plan for HIVRelated Research, 2013.

[17] M. B. Klein, K. C. Rollet, S. Saeed et al., "HIV and hepatitis C virus coinfection in Canada: challenges and opportunities for reducing preventable morbidity and mortality," HIV Medicine, vol. 14, no. 1, pp. 10-20, 2013.

[18] D. Goldberg, J. D. Lewis, S. D. Halpern, M. Weiner, and V. Lo Re III, "Validation of three coding algorithms to identify patients with end-stage liver disease in an administrative database," Pharmacoepidemiology and Drug Safety, vol. 21, no. 7, pp. 765769, 2012.

[19] C. McAdam-Marx, L. J. McGarry, C. A. Hane, J. Biskupiak, B. Deniz, and D. I. Brixner, "All-Cause and incremental per patient per year cost associated with chronic hepatitis $\mathrm{C}$ virus and associated liver complications in the United States: a managed care perspective," Journal of Managed Care Pharmacy, vol. 17, no. 7, pp. 531-546, 2011.

[20] A. Lifson, W. Belloso, R. Davey et al., "Development of diagnostic criteria for serious non-AIDS events in HIV clinical trials," HIV Clinical Trials, vol. 11, no. 4, pp. 205-219, 2010.

[21] E. Rosenthal, D. Salmon-Céron, C. Lewden et al., "Liver-related deaths in HIV-infected patients between 1995 and 2005 in the French GERMIVIC Joint Study Group Network (Mortavic 2005 Study in collaboration with the Mortalité 2005 survey, ANRS EN19)," HIV Medicine, vol. 10, no. 5, pp. 282-289, 2009.

[22] M. V. Ragni, M. A. Nalesnik, R. Schillo, and Q. Dang, "Highly active antiretroviral therapy improves ESLD-free survival in HIV-HCV co-infection," Haemophilia, vol. 15, no. 2, pp. 552558, 2009.

[23] J. Murillas, A. Rimola, M. Laguno et al., "The model for endstage liver disease score is the best prognostic factor in human immunodeficiency virus 1-infected patients with end-stage liver disease: a prospective cohort study," Liver Transplantation, vol. 15, no. 9, pp. 1133-1141, 2009.

[24] J. Baillargeon, R. D. Soloway, D. Paar et al., "End-stage liver disease in a state prison population," Annals of Epidemiology, vol. 17, no. 10, pp. 808-813, 2007.

[25] N. Merchante, J. A. Girón-González, M. González-Serrano et al., "Survival and prognostic factors of HIV-infected patients with HCV-related end-stage liver disease," AIDS, vol. 20, no. 1, pp. 49-57, 2006.

[26] J. J. Goedert, A. Hatzakis, K. E. Sherman, and M. E. Eyster, "Lack of association of hepatitis $\mathrm{C}$ virus load and genotype with risk of end-stage liver disease in patients with human immunodeficiency virus coinfection," Journal of Infectious Diseases, vol. 184, no. 9, pp. 1202-1205, 2001.

[27] M. V. Ragni and S. H. Belle, "Impact of human immunodeficiency virus infection on progression to end-stage liver disease in individuals with hemophilia and hepatitis C virus infection," Journal of Infectious Diseases, vol. 183, no. 7, pp. 1112-1115, 2001.

[28] V. Jotwani, Y. Li, C. Grunfeld, A. I. Choi, and M. G. Shlipak, "Risk factors for ESRD in HIV-infected individuals: traditional and HIV-related factors," The American Journal of Kidney Diseases, vol. 59, no. 5, pp. 628-635, 2012.

[29] A. I. Choi, Y. Li, C. Parikh, P. A. Volberding, and M. G. Shlipak, "Long-term clinical consequences of acute kidney injury in the HIV-infected," Kidney International, vol. 78, no. 5, pp. 478-485, 2010.
[30] A. I. Choi, R. A. Rodriguez, P. Bacchetti, D. Bertenthal, P. A. Volberding, and A. M. O'Hare, "Racial differences in end-stage renal disease rates in HIV infection versus diabetes," Journal of the American Society of Nephrology, vol. 18, no. 11, pp. 29682974, 2007.

[31] G. M. Lucas, B. Lau, M. G. Atta, D. M. Fine, J. Keruly, and R. D. Moore, "Chronic kidney disease incidence, and progression to end-stage renal disease, in HIV-infected individuals: a tale of two races," Journal of Infectious Diseases, vol. 197, no. 11, pp. $1548-1557,2008$.

[32] S. K. Gupta, J. A. Eustace, J. A. Winston et al., "Guidelines for the management of chronic kidney disease in HIV-infected patients: recommendations of the HIV Medicine Association of the Infectious Diseases Society of America," Clinical Infectious Diseases, vol. 40, no. 11, pp. 1559-1585, 2005.

[33] T. Brown, S. Cole, X. Li et al., "Antiretroviral therapy and the prevalence and incidence of diabetes mellitus in the multicenter AIDS cohort study," Archives of Internal Medicine, vol. 165, no. 10, pp. 1179-1184, 2005.

[34] E. C. Seaberg, A. Muñoz, M. Lu et al., "Association between highly active antiretroviral therapy and hypertension in a large cohort of men followed from 1984 to 2003," AIDS, vol. 19, no. 9, pp. 953-960, 2005.

[35] J. Tsui, E. Vittinghoff, K. Anastos et al., "Hepatitis C seropositivity and kidney function decline among women with HIV: data from the Women's Interagency HIV Study," American Journal of Kidney Diseases, vol. 54, no. 1, pp. 43-50, 2009.

[36] S. E. Kellerman, D. L. Hanson, A. D. McNaghten, and P. L. Fleming, "Prevalence of chronic hepatitis B and incidence of acute hepatitis B infection in human immunodeficiency virusinfected subjects," The Journal of Infectious Diseases, vol. 188, no. 4, pp. 571-577, 2003.

[37] M. L. Rodríguez-Méndez, A. González-Quintela, A. Aguilera, and E. Barrio, "Prevalence, patterns, and course of past hepatitis $B$ virus infection in intravenous drug users with HIV-1 infection," The American Journal of Gastroenterology, vol. 95, no. 5, pp. 1316-1322, 2000.

[38] B. H. McGovern, "The epidemiology, natural history and prevention of hepatitis B: implications of HIV coinfection," Antiviral Therapy, vol. 12, supplement 3, pp. H3-H13, 2007.

[39] C. T. Staples Jr., D. Rimland, and D. Dudas, "Hepatitis C in the HIV (human immunodeficiency virus) Atlanta V.A. (Veterans Affairs Medical Center) Cohort Study (HAVACS): the effect of coinfection on survival," Clinical Infectious Diseases, vol. 29, no. 1, pp. 150-154, 1999.

[40] K. E. Sherman, S. D. Rouster, R. T. Chung, and N. Rajicic, "Hepatitis C virus prevalence among patients infected with human immunodefidency virus: a cross-sectional analysis of the US Adult AIDS Clinical Trials Group," Clinical Infectious Diseases, vol. 34, no. 6, pp. 831-837, 2002.

[41] G. Chander, J. Josephs, J. A. Fleishman et al., "Alcohol use among HIV-infected persons in care: results of a multi-site survey," HIV Medicine, vol. 9, no. 4, pp. 196-202, 2008.

[42] G. Chander, B. Lau, and R. D. Moore, "Hazardous alcohol use: a risk factor for non-adherence and lack of suppression in HIV infection," Journal of Acquired Immune Deficiency Syndromes, vol. 43, no. 4, pp. 411-417, 2006.

[43] J. Thorpe, S. Saeed, E. E. M. Moodie, and M. B. Klein, "Antiretroviral treatment interruption leads to progression of liver fibrosis in HIV-hepatitis C virus co-infection," AIDS, vol. 25, no. 7, pp. 967-975, 2011. 
[44] M. López-Diéguez, M. L. Montes, J. F. Pascual-Pareja et al., "The natural history of liver cirrhosis in HIV-hepatitis C viruscoinfected patients," AIDS, vol. 25, no. 7, pp. 899-904, 2011.

[45] B. N. Limketkai, S. H. Mehta, C. G. Sutcliffe et al., "Relationship of liver disease stage and antiviral therapy with liver-related events and death in adults coinfected with HIV/HCV," The Journal of the American Medical Association, vol. 308, no. 4, pp. 370-378, 2012.

[46] V. L. Re, J. K. Lim, M. B. Goetz et al., "Validity of diagnostic codes and liver-related laboratory abnormalities to identify hepatic decompensation events in the Veterans Aging Cohort Study," Pharmacoepidemiology and Drug Safety, vol. 20, no. 7, pp. 689-699, 2011.

[47] H. M. Crane, S. R. Heckbert, D. R. Drozd et al., "Lessons learned from the design and implementation of myocardial infarction adjudication tailored for HIV clinical cohorts," American Journal of Epidemiology, vol. 179, no. 8, pp. 996-1005, 2014.

[48] S. J. Gange, M. M. Kitahata, M. S. Saag et al., "Cohort profile: the North American AIDS cohort collaboration on research and design (NA-ACCORD)," International Journal of Epidemiology, vol. 36, no. 2, pp. 294-301, 2007.

[49] H. M. Crane, B. Kestenbaum, R. D. Harrington, and M. M. Kitahata, "Amprenavir and didanosine are associated with declining kidney function among patients receiving tenofovir," AIDS, vol. 21, no. 11, pp. 1431-1439, 2007.

[50] A. S. Levey, L. A. Stevens, C. H. Schmid et al., "A new equation to estimate glomerular filtration rate," Annals of Internal Medicine, vol. 150, no. 9, pp. 604-612, 2009.

[51] C.-T. Wai, J. K. Greenson, R. J. Fontana et al., "A simple noninvasive index can predict both significant fibrosis and cirrhosis in patients with chronic hepatitis C," Hepatology, vol. 38, no. 2, pp. 518-526, 2003.

[52] R. K. Sterling, E. Lissen, N. Clumeck et al., "Development of a simple noninvasive index to predict significant fibrosis in patients with HIV/HCV coinfection," Hepatology, vol. 43, no. 6, pp. 1317-1325, 2006.

[53] W. E. Barlow, L. Ichikawa, D. Rosner, and S. Izumi, "Analysis of case-cohort designs," Journal of Clinical Epidemiology, vol. 52, no. 12, pp. 1165-1172, 1999.

[54] M. M. Kitahata, B. Rodriguez, R. Haubrich et al., "Cohort profile: the centers for AIDS research network of integrated clinical systems," International Journal of Epidemiology, vol. 37, no. 5, pp. 948-955, 2008. 


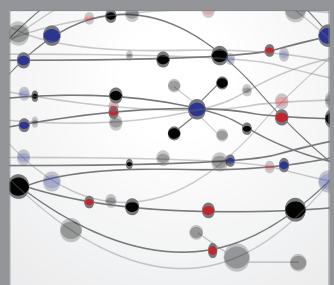

The Scientific World Journal
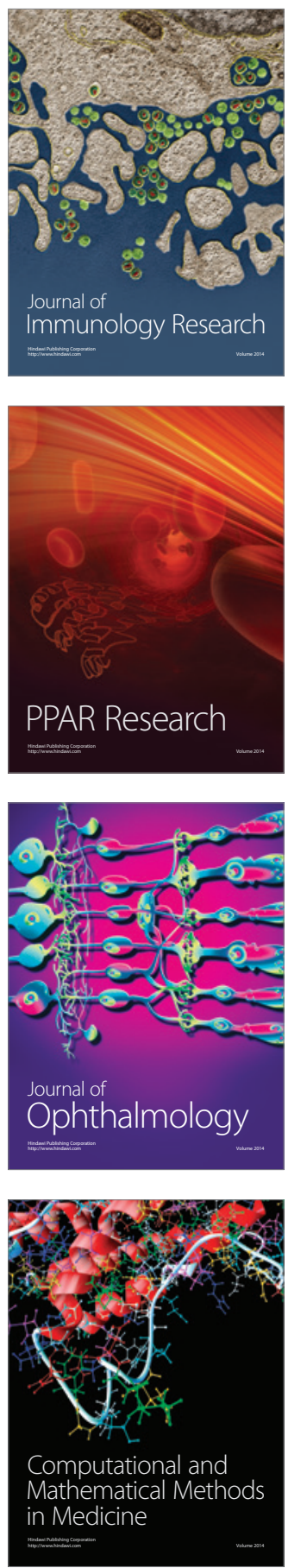

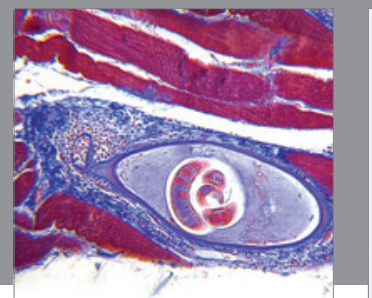

Gastroenterology

Research and Practice
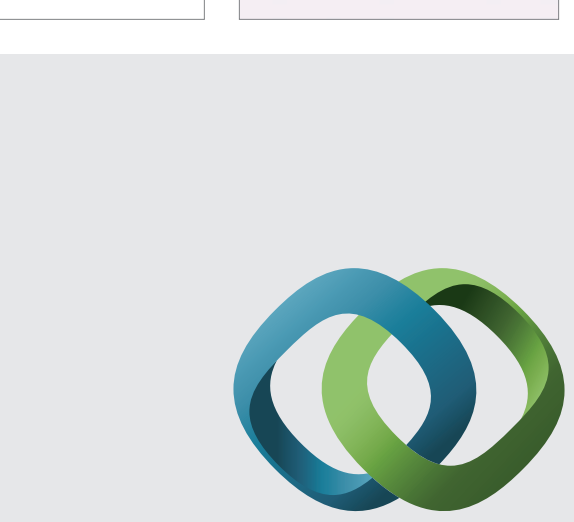

\section{Hindawi}

Submit your manuscripts at

http://www.hindawi.com
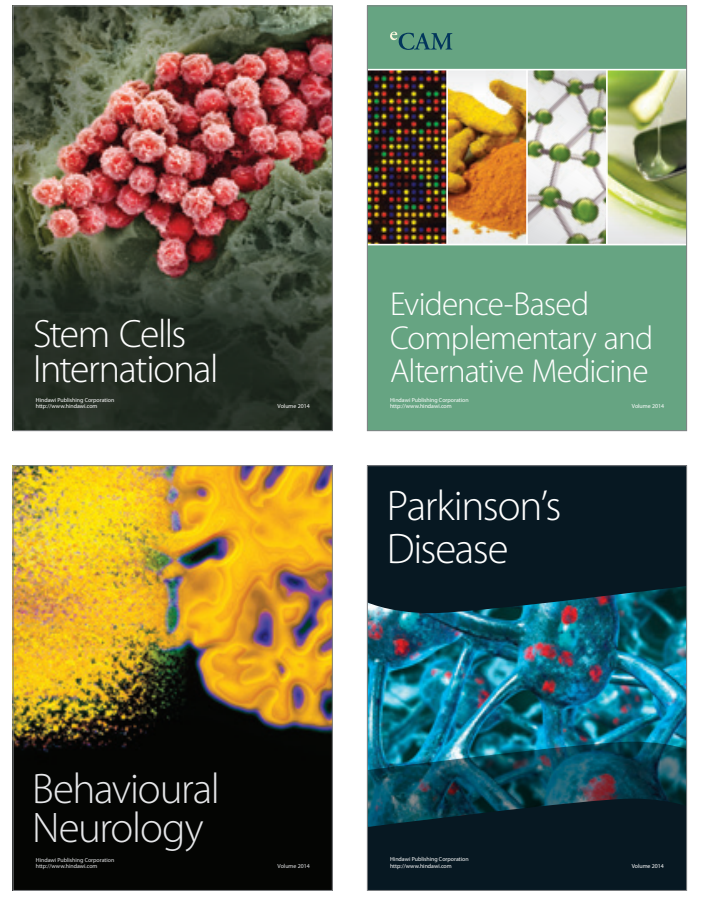
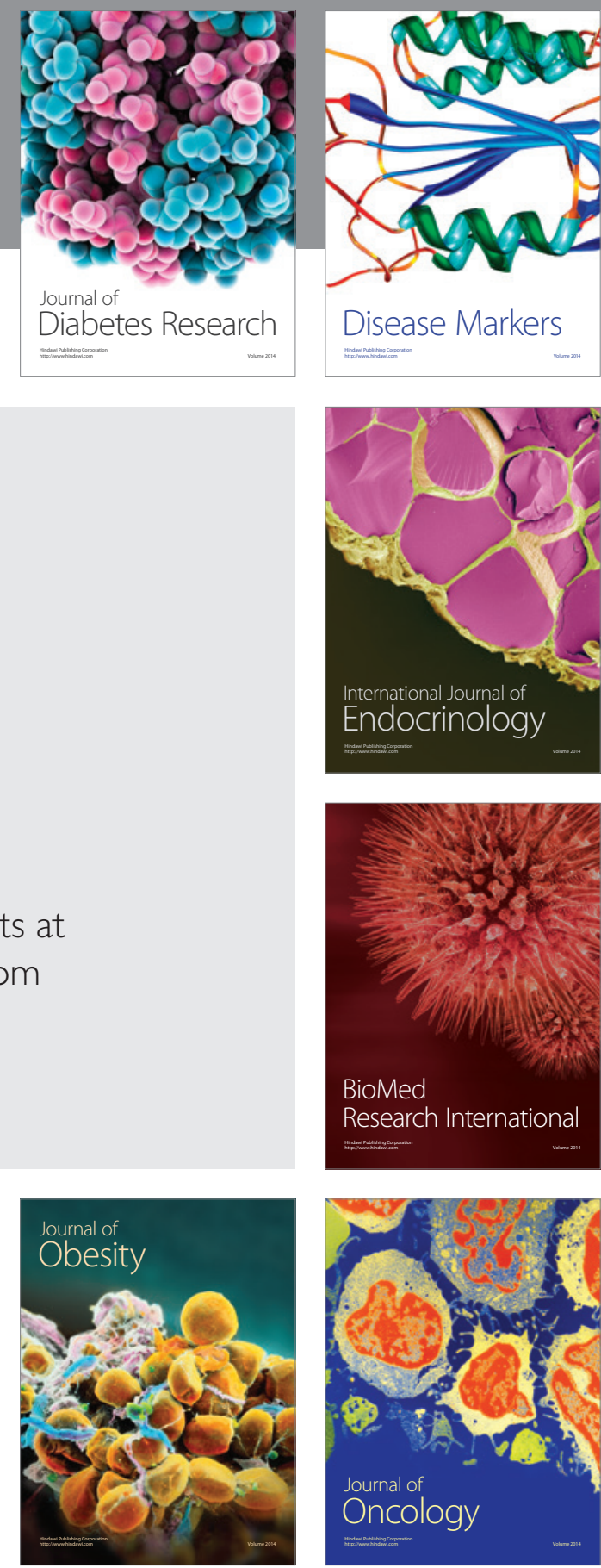

Disease Markers
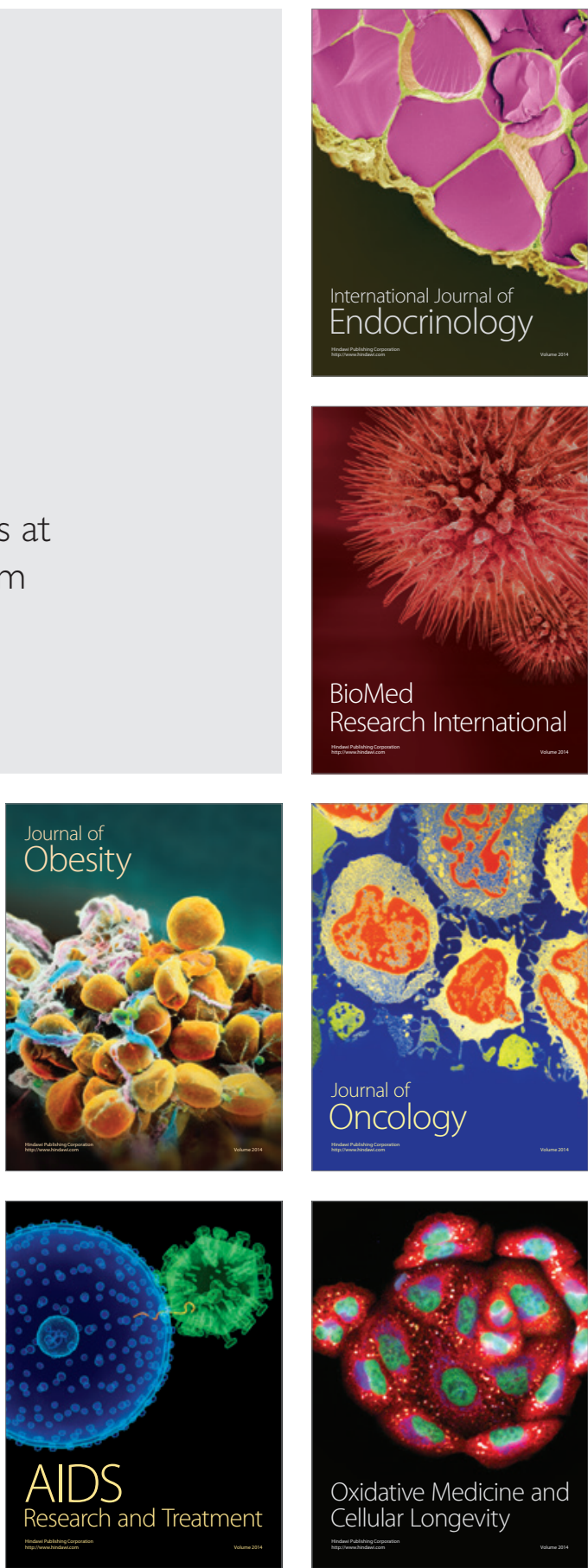